\title{
A Tractable Line-of-Sight Product Channel Model: Application to Wireless Powered Communications
}

\author{
F. J. Lopez-Martinez*, L. Moreno-Pozas*, U. Fernandez-Plazaola*, \\ J. F. Paris*, E. Martos-Naya*, J. M. Romero-Jerez ${ }^{+}$ \\ ${ }^{*}$ Dpto. de Ingeniería de Comunicaciones, ETSI Telecomunicacion \\ ${ }^{+}$Dpto. de Tecnología Electrónica, ETSI Telecomunicacion \\ Universidad de Málaga, Málaga, E-29071, Spain \\ email: \{fjlopezm,lmp,unai,paris,eduardo\}@ic.uma.es, romero@dte.uma.es.
}

\begin{abstract}
We here present a general and tractable fading model for line-of-sight (LOS) scenarios, which is based on the product of two independent and non-identically distributed $\kappa$ $\mu$ shadowed random variables. Simple closed-form expressions for the probability density function and cumulative distribution function are derived, which are as tractable as the corresponding expressions derived from a product of Nakagami- $m$ random variables. This newly proposed model simplifies the challenging characterization of LOS product channels, as well as combinations of LOS channels with non-LOS ones. Results are used to analyze performance measures of interest in the context of wireless powered communications.
\end{abstract}

\section{INTRODUCTION}

In wireless communications, the so-called product channel $Z=X Y$ is related to the product of two random processes $X$ and $Y$ associated to the stochastic nature of two wireless communication channels. The product channel naturally arises in the context of communication systems assisted by relays [1], when modeling propagation effects such as keyholes [2], diffraction [3] and composite fading [4], or turbulence-induced scintillation in free-space optical communications [5]. Further scenarios on which the product channel characterization is essential also include wireless powered communications (WPC) [6-8]. Thus, the statistical characterization of the product channel is of paramount relevance for understanding the performance limits of wireless communication systems operating in these scenarios, either in line-of-sight (LOS) or non-LOS (NLOS) propagation conditions.

The characterization of LOS product (or LOSxLOS) channels is very challenging and cannot be given in closed-form, but in terms of double infinite sums of special functions $[9,10]$. Therefore, for the sake of tractability, some authors have approximated both LOS links as NLOS ones, which have closed-form characterization [1, 3]. Indeed, using the Nakagami- $m$ distribution for approximating the Rician distribution is a classical approach which can simplify the characterization of some scenarios $[11,12]$. In other scenarios, however, despite being simple and insightful, the results derived therein can give inaccurate approximations when the propagation conditions are clearly LOS. Moreover, the approximation of the Rician distribution through a Nakagami- $m$ distribution has severe limitations related to the different diversity order of such distributions [13].
With all these considerations, the literature is lacking from LOS product channel models which are analytically tractable. Since the complexity of previous results for LOS product channel models are mainly due to the challenge posed by considering a product of two Rician random variables (RVs), we here propose to characterize product channels by means of a more general distribution, which in turn will help simplifying the problem. We will introduce a product channel model based on the $\kappa-\mu$ shadowed fading distribution $[14,15]$, built as the product of two independent and non-identically distributed (INID) $\kappa-\mu$ shadowed RVs with integer fading parameters, which includes the Rician product model as a special case. For the sake of shorthand notation, we will refer to this new fading distribution as the $\mathcal{P}$-distribution (where $\mathcal{P}$ stands for product). Although we here focus on simplifying the analysis of Rician product channels, the results here presented for the $\kappa-\mu$ shadowed product channel can unify the analysis of a vast set of product models as special cases, including LOSxLOS product channel based on the $\kappa$ - $\mu$ distribution, as well as LOSXNLOS and NLOSxNLOS product channels based on the Nakagami- $m$ and Rayleigh distributions [16]. The usefulness of this new distribution is exemplified in the context of wireless powered communications. Specifically, we investigate the average throughput in a WPC setup on which all links are inherently LOS.

\section{Statistical Characterization}

\section{A. Preliminary results}

Throughout this paper, we will consider the distributions associated to the power envelope in $\kappa$ - $\mu$ shadowed fading channels (or equivalently, the instantaneous receive SNR $\gamma$ ).

Lemma 1 (The $\kappa$ - $\mu$ shadowed distribution with integer fading parameters [17]): Let $\gamma$ be a $\kappa-\mu$ shadowed random variable with mean $\bar{\gamma}$ and shape parameters $\kappa, \mu$ and $m$ [14]. If the parameters $\mu$ and $m$ are positive integers, then for any arbitrary non-negative real $\kappa$ the probability density function (PDF) and cumulative distribution function (CDF) of $\gamma$ are given by [17, eq. (4-10)] 


$$
\begin{gathered}
f_{\mathcal{S}}(x)=\sum_{j=0}^{M} C_{j} \underbrace{\frac{x^{m_{j}-1} e^{-\frac{x}{\Omega_{j}}}}{\Omega_{j}^{m_{j}}\left(m_{j}-1\right) !}}_{f_{\mathcal{K}}\left(\Omega_{j} ; m_{j} ; x\right)} \\
F_{\mathcal{S}}(x)=1-\sum_{j=0}^{M} C_{j} e^{-\frac{x}{\Omega_{j}}} \sum_{r=0}^{m_{j}-1} \frac{1}{r !}\left(\frac{x}{\Omega_{j}}\right)^{r}
\end{gathered}
$$

where $M$ and the set of parameters $\left\{C_{j}, m_{j}, \Omega_{j}\right\}_{j=1, \ldots, M}$ are expressed in terms of $\bar{\gamma}, \kappa, \mu$ and $m$ according to Table I. In (1) $f_{\mathcal{K}}(\cdot)$ represents the PDF of a squared Nakagami- $m$ distribution, (i.e. a Gamma distribution).

According to Lemma 1, the $\kappa-\mu$ shadowed distribution with integer fading parameters $m$ and $\mu$ can be expressed as a finite mixture of squared Nakagami- $m$ (or Gamma) distribution. To theoretically obtain the Rician distribution as special case, we need to set $\mu=1$ and tend $m \rightarrow \infty$. However, in practice, the $\kappa-\mu$ shadowed distribution converges rapidly to the Rician distribution, i.e. for $m \approx 15-20$ [17].

Corollary 1 (Product of Two Squared Nakagami-m RVs): Let $Z=W \hat{W}$ be the product of two INID squared Nakagami$m$ random variables $W$ and $\hat{W}$ with means $\Omega$ and $\hat{\Omega}$, where the corresponding shape parameters $m$ and $\hat{m}$ are arbitrary positive integer numbers. Then, the corresponding PDF and $\mathrm{CDF}$ are given by

$$
\begin{gathered}
f_{\Gamma \Gamma}(x)=\frac{2 x^{\frac{m+\hat{m}}{2}-1}}{\Gamma(m) \Gamma(\hat{m})(\Omega \hat{\Omega})^{\frac{m+\hat{m}}{2}}} K_{m-\hat{m}}\left(\sqrt{\frac{4 x}{\Omega \hat{\Omega}}}\right), \\
F_{\Gamma \Gamma}(x)=1-\sum_{k=0}^{m-1} \frac{2}{k ! \Gamma(\hat{m})}\left(\frac{x}{\Omega \hat{\Omega}}\right)^{\frac{k+\hat{m}}{2}} K_{\hat{m}-k}\left(\sqrt{\frac{4 x}{\Omega \hat{\Omega}}}\right)
\end{gathered}
$$

where $K_{\nu}$ is the modified Bessel function of the second kind, and $\Gamma(\cdot)$ is the Gamma function.

Proof: The PDF follows from the corresponding expression given in [1] after performing a simple random variable transformation of the type $Y=X^{2}$. The CDF follows from [7, eq. 8] by specializing the parameter $a=1$.

The distribution described in Corollary 1 is essentially a Gamma-Gamma (ГГ) distribution, up to a trivial re-scaling by $\Omega$. For the sake of notation simplicity, in this work we will refer to this distribution as a $Г \Gamma$ distribution.

\section{B. Main results}

By means of the previous results and considerations, we will now characterize the distribution of the product of two INID $\kappa-\mu$ shadowed fading variables with integer fading parameters.

Proposition 1 (The $\mathcal{P}$-distribution as a finite mixture of $\Gamma \Gamma$ distributions): Let $Z$ be the product of two INID squared $\kappa-\mu$ shadowed random variables $X$ and $\hat{X}$ with means $\bar{\gamma}$ and $\widetilde{\gamma}$. The corresponding shape parameters $\kappa$ and $\hat{\kappa}$ are arbitrary nonnegative real numbers and the remainder shape parameters $\mu$ and $m$ for $X$, and $\hat{\mu}$ and $\hat{m}$ for $\hat{X}$ are positive integers. Under these conditions, $Z=X \hat{X}$ is distributed as a $\Gamma \Gamma$ finite mixture with the following PDF

$$
\begin{aligned}
& f_{Z}(z)=\sum_{j=0}^{M} \sum_{h=0}^{\hat{M}} C_{j} \hat{C}_{h} \times \\
& \underbrace{\frac{2 z^{\frac{m_{j}+\hat{m}_{h}}{2}}-1}{\Gamma\left(m_{j}\right) \Gamma\left(\hat{m}_{h}\right)\left(\Omega_{j} \Omega_{h}\right)^{\frac{m_{j}+\hat{m}_{h}}{2}}} K_{m_{j}-\hat{m}_{h}}\left(\sqrt{\frac{4 z}{\Omega_{j} \hat{\Omega}_{h}}}\right)}_{f_{\Gamma \Gamma}\left(z ;\left\{\Omega_{j}, m_{j}\right\} ;\left\{\hat{\Omega}_{h}, \hat{m}_{h}\right\}\right)}
\end{aligned}
$$

where the parameters $M$ and $\left\{C_{j}, m_{j}, \Omega_{j}\right\}_{j=1, \ldots, M}$ are expressed in terms of $\bar{\gamma}, \kappa, \mu$ and $m$ according to Table 1; similarly the parameters $\hat{M}$ and $\left\{\hat{C}_{h}, \hat{m}_{h}, \hat{\Omega}_{h}\right\}_{h=1, \ldots, \hat{M}}$ are also expressed in terms of $\widetilde{\gamma}, \hat{\kappa}, \hat{\mu}$ and $\hat{m}$.

Proof: The MGF of $Z$ can be computed as

$$
\begin{aligned}
& \mathcal{M}_{Z}(s) \triangleq E\left[e^{X \hat{X} s}\right]= \\
& \int_{0}^{\infty} E\left[e^{x \hat{X} s} \mid X=x\right] f_{X}(x) d x=\int_{0}^{\infty} f_{X}(x) \mathcal{M}_{\hat{X}}(s x) d x .
\end{aligned}
$$

Now, according to Corollary 1 the MGF of $\hat{X}$ in (6) can be expressed in terms of the squared Nakagami- $m$ MGF $\mathcal{M}_{\mathcal{K}}$ as

$$
\mathcal{M}_{\hat{X}}(s x)=\sum_{h=0}^{\hat{M}} \hat{C}_{h} \mathcal{M}_{\mathcal{K}}\left(\hat{\Omega}_{h} ; \hat{m}_{h} ; s x\right) .
$$

Using (1) and (7) in (6) and expanding the integrand yields

$$
\begin{aligned}
& \mathcal{M}_{Z}(s)=\sum_{j=0}^{M} \sum_{h=0}^{\hat{M}} C_{j} \hat{C}_{h} \times \\
& \underbrace{\int_{0}^{\infty} f_{\mathcal{K}}\left(\Omega_{j} ; m_{j} ; x\right) \mathcal{M}_{\mathcal{K}}\left(\hat{\Omega}_{h} ; \hat{m}_{h} ; s x\right) d x}_{\mathcal{M}_{\Gamma \Gamma}\left(s ;\left\{\Omega_{j}, m_{j}\right\} ;\left\{\hat{\Omega}_{h}, \hat{m}_{h}\right\}\right)}
\end{aligned}
$$

where $\mathcal{M}_{\Gamma \Gamma}\left(s ;\left\{\Omega_{j}, m_{j}\right\} ;\left\{\hat{\Omega}_{h}, \hat{m}_{h}\right\}\right)$ is the MGF of the $Г \Gamma$. Thus, applying the inverse Laplace Transform in (8) and considering (3) completes the proof.

Proposition 1 states that the $\mathcal{P}$-distribution can be expressed in closed-form as a finite sum of well-known special functions. Since the $\mathcal{P}$-distribution is more general and simpler than the Rician product distribution (which is but a special case for $\mu=\hat{\mu}=1$ and sufficiently large $m$ and $\hat{m}$ ), we advocate for its use as the reference product channel model in a communication-theoretic context. This will be later supported by both theoretical and practical evidences in different scenarios of interest.

Proposition 2 (CDF of the $\mathcal{P}$ distribution as a finite mixture): Let $Z$ be the product of two INID squared $\kappa-\mu$ shadowed random variables $X$ and $\hat{X}$ with means $\bar{\gamma}$ and $\widetilde{\gamma}$. The corresponding shape parameters $\kappa$ and $\hat{\kappa}$ are arbitrary nonnegative real numbers and the remainder shape parameters $\mu$ and $m$ for $X$, and $\hat{\mu}$ and $\hat{m}$ for $\hat{X}$ are positive integers. Under these conditions $Z=X \hat{X}$ has the following $\mathrm{CDF}$ 
TABLE I: Parameter values for the $\kappa-\mu$ shadowed distribution with integer $\mu$ and $m$,

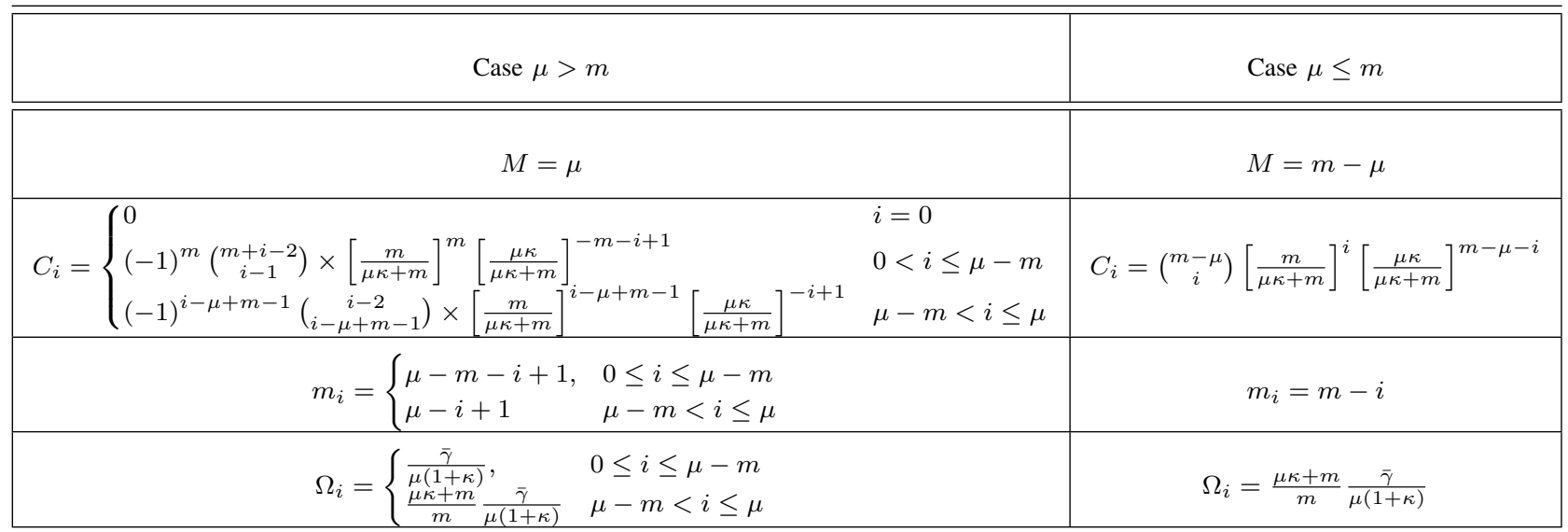

$$
\begin{aligned}
& F_{Z}(z)=\sum_{j=0}^{M} \sum_{h=0}^{\hat{M}} C_{j} \hat{C}_{h} \times \\
& \underbrace{\left[1-\sum_{k=0}^{m_{j}-1} \frac{2}{k ! \Gamma\left(\hat{m}_{h}\right)}\left(\frac{z}{\Omega_{j} \hat{\Omega}_{h}}\right)^{\frac{k+\hat{m}_{h}}{2}} \times K_{\hat{m}_{h}-k}\left(\sqrt{\frac{4 z}{\Omega_{j} \hat{\Omega}_{h}}}\right)\right]}_{F_{\Gamma \Gamma}\left(z ;\left\{\Omega_{j}, m_{j}\right\} ;\left\{\hat{\Omega}_{h}, \hat{m}_{h}\right\}\right)}
\end{aligned}
$$

where the parameters $M$ and $\left\{C_{j}, m_{j}, \Omega_{j}\right\}_{j=1, \ldots, M}, \hat{M}$ and $\left\{\hat{C}_{h}, \hat{m}_{h}, \hat{\Omega}_{h}\right\}_{h=1, \ldots, \hat{M}}$ are those indicated in Proposition 1 .

Proof: The CDF can be derived from the PDF such as

$$
F_{Z}(z)=\int_{0}^{z} f_{Z}(t) d t
$$

From Proposition 1, we have

$$
F_{Z}(z)=\sum_{j=0}^{M} \sum_{h=0}^{\hat{M}} C_{j} \hat{C}_{h} F_{\Gamma \Gamma}\left(z ;\left\{\Omega_{j}, m_{j}\right\} ;\left\{\hat{\Omega}_{h}, \hat{m}_{h}\right\}\right)
$$

where $F_{\Gamma \Gamma}(\cdot)$ is given by equation (4).

Thus, the CDF of the $\mathcal{P}$-distribution is also given in a simple closed-form. This expression has important relevance in practice, since the CDF of the product of INID Rician RVs has a very complicated form, which involves a double infinite sum of Meijer $G$-functions [10, eq. (23)]. Here, the $\mathcal{P}$-distribution function, which includes the Rician product distribution function as a special case, is only given in terms of finite sums of modified Bessel functions of the second kind.

\section{WIRELESS POWERED COMMUNICATIONS}

\section{A. System Model}

Let us consider the following scenario [6,7], on which a source $\mathrm{S}$ communicates with a destination $\mathrm{D}$ with the help of dedicated power beacons (PBs) that wirelessly transfer energy to $\mathrm{S}$. Both $\mathrm{S}$ and $\mathrm{D}$ are equipped with a single antenna, while PBs are equipped with $N$ antennas. The system operation follows a harvest-then-transmit-like policy for every time transmission interval $T$, as follows: during the first $\tau T$ seconds (with $0<\tau<1$ ), the source $\mathrm{S}$ harvests energy from the PBs. During the energy harvesting phase, the received signal at $\mathrm{S}$ can be expressed as

$$
y_{S}=\sqrt{\frac{P}{d_{1}^{\alpha}}} \mathbf{h} \mathbf{x}_{S}+n_{S}
$$

where $P$ is the transmit power at the $\mathrm{PB}, d_{1}$ denotes the distance between $\mathrm{PB}$ and $\mathrm{S}, \alpha$ is the path loss exponent, $\mathbf{h}$ and $\mathbf{x}_{S}$, are $N$ elements vectors denoting the channel response and the transmitted symbols, respectively, and $n_{S}$ is the additive white Gaussian noise (AWGN) with variance $N_{0}$. The elements of $\mathbf{h}=\left[h_{i}\right](i=1 \ldots N)$ are assumed to be independent and identically distributed (IID) with unitary variance. Assuming $\mathbf{x}_{S}$ is formed with optimal beamforming and $n_{S}$ can be neglected during the harvesting phase, the total energy received at the end of the first phase is [6, eq. (5)]

$$
E_{n}=\frac{\eta\|\mathbf{h}\|^{2} P \tau T}{d_{1}^{\alpha}}
$$

where $0<\eta<1$ is the energy conversion efficiency.

In the second phase, $\mathrm{S}$ transmits information to $\mathrm{D}$ using the energy harvested in the first phase during $(1-\tau) T$ seconds. Hence, the received signal $y_{D}$ at $\mathrm{D}$ is given by

$$
y_{D}=\sqrt{\frac{E_{n}}{(1-\tau) T d_{2}^{\alpha}}} g s_{0}+n_{d}
$$

where $d_{2}$ denotes the distance between $\mathrm{S}$ and $\mathrm{D}, g$ is the channel response following an arbitrary fading distribution with unit variance, $s_{0}$ is the information symbol with unit energy, and $n_{d}$ is AWGN with variance $N_{0}$. Therefore, the instantaneous end-to-end SNR can be computed as

$$
\gamma=\frac{\tau \eta\|\mathbf{h}\|^{2}|g|^{2} P}{(1-\tau) d_{1}^{\alpha} d_{2}^{\alpha} N_{0}}
$$

\section{B. Performance analysis}

Direct inspection of (15) reveals that the distribution of $\gamma$ is that of the product of $\|\mathbf{h}\|^{2}$ and $|g|^{2}$, which is ultimately related to the distribution of the product of the underlying 
fading channels between PBs and $\mathrm{S}$, and between $\mathrm{S}$ and $\mathrm{D}$. As argued in [6], the link between PBs and $S$ is inherently LOS because of the relatively short distance between both agents. However, the consideration of the Rician distribution to model the small-scale fading in the PBs-S link is associated to a larger mathematical complexity. For this reason, the Rician distribution was approximated by the Nakagami- $m$ distribution in [6], with $m=(1+K)^{2} /(1+2 K)$. In turn, the S-D link will be NLOS or LOS depending on the specific set-up: both situations were addressed in [6] and [7] by resorting to Rayleigh and Nakagami- $m$ fading, respectively.

In the most general situation, both the PBs-S and the S-D links can be LOS, and therefore the product channel associated to LOS scenarios is the natural choice for characterizing the behavior of the end-to-end SNR. We here propose the use of the $\mathcal{P}$-distribution introduced in Section II for this application, as a workaround to characterize the distribution of $\gamma$ when the Rician distribution is considered. Because $\|\mathbf{h}\|^{2}$ can be expressed as the sum of $N$ squared Rician random variables (i.e. a $\kappa$ - $\mu$ distribution with $\kappa=K$ and $\mu=N$ ), and assuming $|g|^{2}$ to be Rician distributed, the distribution of $\gamma$ is that of the product of $\kappa-\mu$ and Rician random variables. Thus, it arises as a special case of the $\mathcal{P}$-distribution. Compared to the approximation in [6], our approach has a number of benefits which can be summarized as follows:

- The Rician shadowed distribution ( $\kappa-\mu$ shadowed distribution with $\mu=1$ ) and the Rician distribution have a diversity order equal to one, as opposed to the Nakagami$m$ distribution, for which the diversity order is $m$. Thus, approximating the Rician distribution by the Rician shadowed distribution does not affect the diversity order.

- In practice, LOS channels will not be purely Rician because of the inherent fluctuation of the LOS component [18]. In fact, the $\kappa$ - $\mu$ shadowed fading model always provides a better fit to real measurements than the Rician fading model alone, just because the latter is a special case of the former. Thus, the $\mathcal{P}$-distribution is not only simpler and more general than the Rician product distribution, but also closer to the real behavior of the fading channel.

With all the above considerations, the performance of WPC systems in LOS scenarios can be easily evaluated when considering the $\mathcal{P}$-distribution. Assuming that $\mathrm{S}$ transmits at a constant rate $R_{c}$, which may be subjected to outage due to fading, the average throughput can be evaluated as

$$
R_{D C}=\left(1-P_{\text {out }}\right) R_{c}(1-\tau)
$$

where $P_{\text {out }}=\operatorname{Pr}\left\{\gamma<\gamma_{\text {th }}\right\}$ is the outage probability, being $\gamma_{\text {th }}$ the minimum SNR required for a reliable communication. As previously stated, the distribution of the product $\|\mathbf{h}\|^{2} \cdot|g|^{2}$ can be modeled as a product of two independent squared $\kappa-\mu$ shadowed variables with a proper choice of parameters. Thus, the outage probability can be obtained from (9) following the same steps in [6] as

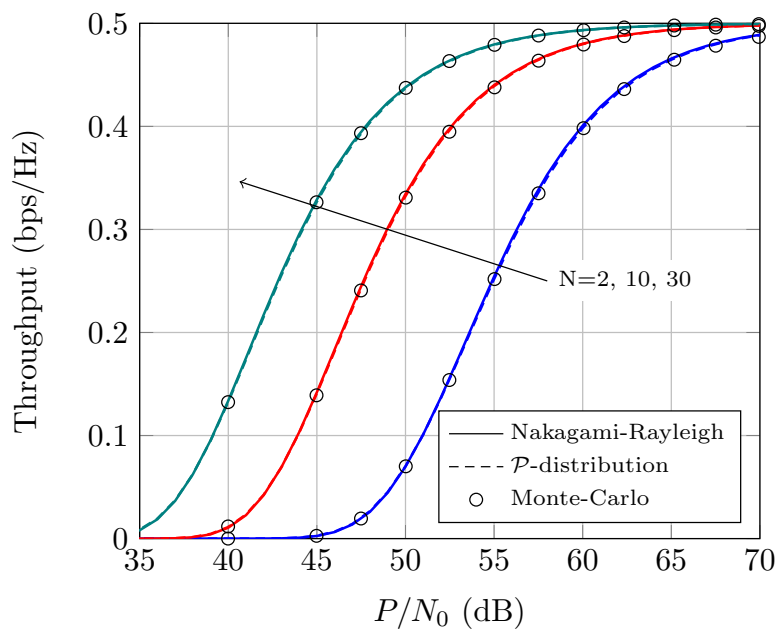

Fig. 1: Average Throughput $R_{D C}$ vs. average SNR, for different values of $N$. LOS $\times$ NLOS scenario. MC simulations correspond to the Rician-Rayleigh case.

$$
P_{\text {out }}=F_{\gamma}\left(\frac{(1-\tau) d_{1}^{\alpha} d_{2}^{\alpha} N_{0}}{\tau \eta P} \gamma_{\text {th }}\right)
$$

\section{NUMERICAL RESULTS}

We now use the results in the previous subsection for evaluating to what extent the Rician product channel can be approximated by a Nakagami- $m$ product channel, in the context of WPC scenarios. The following set of parameters is considered, in coherence with those used in [6]: $R_{c}=1$ $\mathrm{bps} / \mathrm{Hz}$, which implies an outage SNR threshold given by $\gamma_{\mathrm{th}}=2^{R_{c}}-1=1$, the harvesting time is set to $50 \%$ of the interval $T$, the energy conversion efficiency is set to $\eta=0.4$, the path loss exponent is set to $\alpha=2.5$, and distances are set to be $d_{1}=8 \mathrm{~m}$ and $d_{2}=15 \mathrm{~m}$, respectively.

In Fig. 1, the throughput obtained from (16) is evaluated as a function of the average SNR, for different numbers of antennas at the PBs. Because of the beamforming strategy used by the PBs, the distribution of $\|\mathbf{h}\|^{2}$ is that of a squared $\kappa-\mu$ random variable. We first assume that the channel between the source $S$ and the destination D is NLOS as in [6], so it can be safely modeled by a Rayleigh fading channel. Thus, we here compare two alternatives for evaluating (16): the first one is approximating $\|\mathbf{h}\|^{2}$ by a squared Nakagami- $m$ (gamma) distribution with $m=(1+K)^{2} /(1+2 K) \cdot N$ as in [6], and then using the statistics of a Nakagami-Rayleigh product channel; the second one is using the $\mathcal{P}$-distribution with $\kappa=$ $K, \mu=N, \hat{\kappa}=0, \hat{\mu}=1$, and sufficiently large $m$ and $\hat{m}$ (i.e. $m=\hat{m}=20$ ). We consider $K=3+\sqrt{12}$ as in [6]. We observe that both approaches yield very similar results for the set of parameters here considered. Thus, the approximation can be safely used for a product channel built from a LOS and a NLOS individual channels, for the evaluation of (16). The average throughput saturates at $R_{D C} \approx R_{c}(1-\tau)$ for sufficiently large SNR.

However, things change when both channels are considered to be LOS. In Fig. 2, the S-D link is also assumed to be LOS with equal $K$ parameter as in Fig. 1. We now see a 


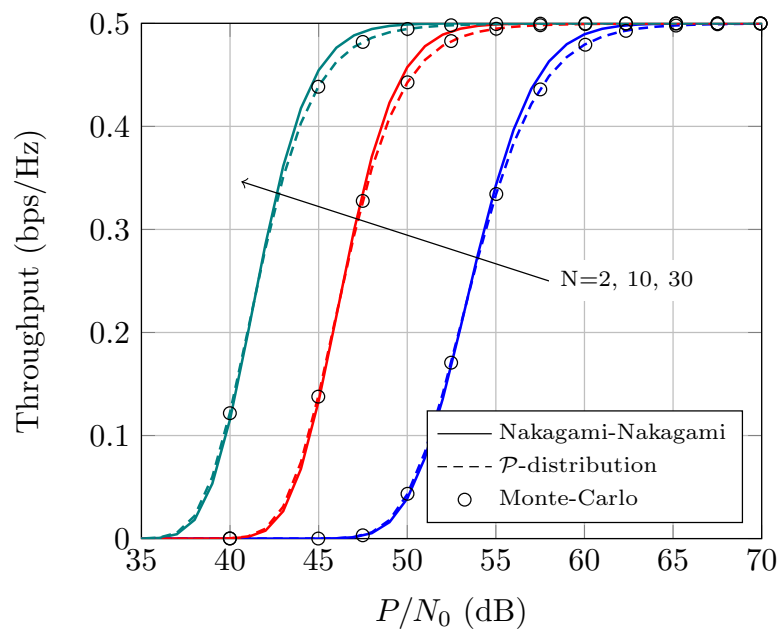

Fig. 2: Average Throughput $R_{D C}$ vs. average SNR, for different values of $N$. LOS $\times$ LOS scenario. MC simulations correspond to the Rician-Rician case.

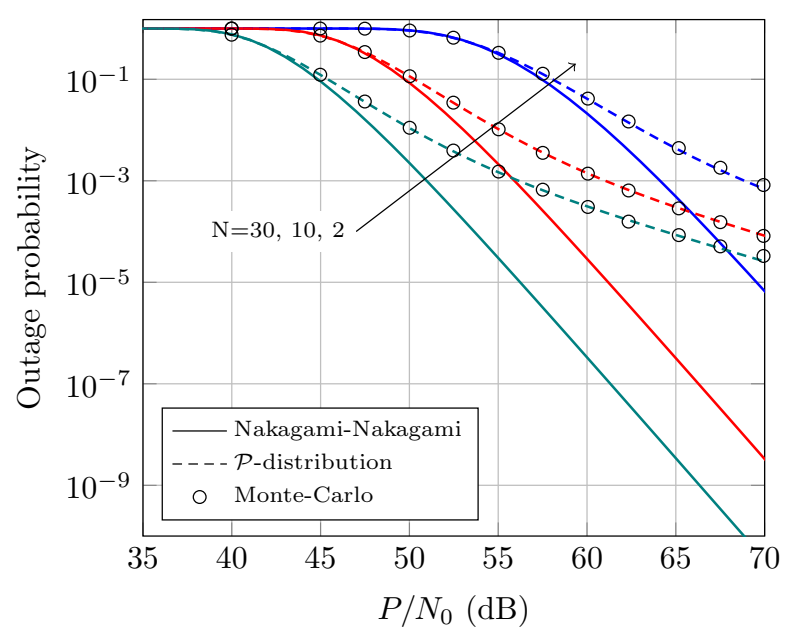

Fig. 3: Outage probability vs. average SNR, for different values of $N$. LOS $\times$ LOS scenario. MC simulations correspond to the Rician-Rician case.

noticeable difference between the approximation in [6] and the exact result using the $\mathcal{P}$-distribution with $\kappa=\hat{\kappa}=K$, $\mu=N, \hat{\mu}=1$ and $m=\hat{m}=20$, which perfectly matches the Monte-Carlo simulations run for the Rician product case. This becomes even more evident when analyzing the tail behavior of the outage probability in Fig. 3, where the different diversity orders of the Nakagami- $m$ distribution and the Rician distribution can be observed. Thus, in this scenario it is not recommended to use the Nakagami- $m$ product channel to approximate the Rician product channel.

\section{Conclusions}

We introduced a new model based on the product of two INID $\kappa-\mu$ shadowed RVs, which has allowed us to characterize LOS product channels models with very simple closed-form expressions. The usefulness of the results has been exemplified through the analysis of wireless powered communications. Specifically, we have observed that previous channel approximations based on the Nakagami- $m$ distribution failed to provide good accuracy for such non-ergodic measures in LOSxLOS scenarios. Moreover, our model allows for simplifying previous exact theoretical results for LOSxLOS and LOSXNLOS product channels.

\section{ACKNOWLEDGMENTS}

This work has been funded by the Spanish Government and the European Fund for Regional Development FEDER (TEC2014-57901-R and TEC2017-87913-R)

\section{REFERENCES}

[1] G. K. Karagiannidis, N. C. Sagias, and P. T. Mathiopoulos, "N*Nakagami: A novel stochastic model for cascaded fading channels," IEEE Trans. Commun., vol. 55, no. 8, pp. 1453-1458, Aug. 2007.

[2] D. Chizhik, G. J. Foschini, M. J. Gans, and R. A. Valenzuela, "Keyholes, correlations, and capacities of multielement transmit and receive antennas," IEEE Trans. Wireless Commun., vol. 1, no. 2, pp. 361-368, Apr. 2002.

[3] V. Erceg, S. J. Fortune, J. Ling, A. J. Rustako, and R. A. Valenzuela, "Comparisons of a computer-based propagation prediction tool with experimental data collected in urban microcellular environments," IEEE J. Sel. Areas Commun., vol. 15, no. 4, pp. 677-684, May 1997.

[4] S. K. Yoo, S. L. Cotton, P. C. Sofotasios, and S. Freear, "Shadowed fading in indoor off-body communication channels: A statistical characterization using the $\kappa-\mu /$ gamma composite fading model," IEEE Trans. Wireless Commun., vol. 15, no. 8, pp. 5231-5244, Aug. 2016.

[5] M. Al-Habash, L. C. Andrews, and R. L. Phillips, "Mathematical model for the irradiance probability density function of a laser beam propagating through turbulent media," Optical Engineering, vol. 40, no. 8, pp. 1554-1562, Aug. 2001.

[6] C. Zhong, X. Chen, Z. Zhang, and G. K. Karagiannidis, "Wirelesspowered communications: Performance analysis and optimization," IEEE Trans. Commun., vol. 63, no. 12, pp. 5178-5190, Dec. 2015.

[7] P.-T. Van, H.-H. N. Le, M.-D. N. Le, and D.-B. Ha, "Performance analysis in wireless power transfer system over Nakagami fading channels," in 2016 International Conference on Electronics, Information, and Communications (ICEIC), Jan 2016.

[8] O. L. Alcaraz-López, H. Alves, R. D. Souza, and E. G. Fernández, "Ultrareliable short-packet communications with wireless energy transfer," IEEE Signal Process. Lett., vol. 24, no. 4, pp. 387-391, Apr. 2017.

[9] N. O'Donoughue and J. M. F. Moura, "On the product of independent complex gaussians," IEEE Trans. Signal Process., vol. 60, no. 3, pp. 1050-1063, Mar. 2012.

[10] A. Bekkali, S. Zou, A. Kadri, M. Crisp, and R. V. Penty, "Performance analysis of passive UHF RFID systems under cascaded fading channels and interference effects," IEEE Trans. Wireless Commun., vol. 14, no. 3, pp. 1421-1433, Mar. 2015.

[11] M. Nakagami, "The m-distribution- a general formula of intensity distribution of rapid fading," Stat. Methods of Radio Propagation, 1960.

[12] M. K. Simon and M.-S. Alouini, Digital Communication over Fading Channels. Wiley-IEEE Press, 2005. [Online]. Available: http://www.worldcat.org/isbn/0471649538

[13] Z. Wang and G. B. Giannakis, "A simple and general parameterization quantifying performance in fading channels," IEEE Trans. Commun., vol. 51, no. 8, pp. 1389-1398, Aug. 2003.

[14] J. F. Paris, "Statistical characterization of $\kappa-\mu$ shadowed fading," IEEE Trans. Veh. Technol., vol. 63, no. 2, pp. 518-526, Feb. 2014.

[15] S. L. Cotton, "Human body shadowing in cellular device-to-device communications: Channel modeling using the shadowed $\kappa-\mu$ fading model," IEEE J. Sel. Areas Commun., vol. 33, no. 1, pp. 111-119, Jan. 2015.

[16] L. Moreno-Pozas, F. J. Lopez-Martinez, J. F. Paris, and E. MartosNaya, "The $\kappa-\mu$ shadowed fading model: Unifying the $\kappa-\mu$ and $\eta-\mu$ distributions," IEEE Trans. Veh. Technol., vol. 65, no. 12, pp. 96309641, Dec. 2016.

[17] F. J. Lopez-Martinez, J. F. Paris, and J. M. Romero-Jerez, "The $\kappa-\mu$ shadowed fading model with integer fading parameters," IEEE Trans. Veh. Technol., vol. 66, no. 9, pp. 7653-7662, Sept. 2017.

[18] J. M. Romero-Jerez, F. J. Lopez-Martinez, J. F. Paris, and A. J. Goldsmith, "The fluctuating two-ray fading model: Statistical characterization and performance analysis," IEEE Trans. Wireless Commun., vol. 16, no. 7, pp. 4420-4432, July 2017. 